\title{
Comunicación: El audiovisual en el aprendizaje de competencias transversales
}

\section{Gloria Domènech Martínez, Francisco Millán Salas y Gloria Domènech Tudela}

Profesora de Derecho Administrativo (Universitat Politècnica de València, glodomar@urb.upv.es), Profesor Contratado Doctor de Derecho Civil (Universidad Complutense de Madrid, fmillans@ucm.es), y Profesora (Institut Torre de Malla. Parets del Vallès. Catalunya, gdomene9@xtec.cat).

\begin{abstract}
One of the changes introduced by the Bologna Declaration produced teaching methodologies that became oriented to evaluate the effort of the student, resulting in greater teacher interaction in their training. Following this line, the Universitat Politècnica de València (UPV), through its strategic UPV2020 plan, accredits the Transversal Competences of its graduates, among which is the CT10 referred to as the Knowledge of Contemporary Problems.

For a graduate with a degree in Audiovisual Communication (GCAV), the audiovisual product is their way of expressing their thoughts and ideas on the political, social and legal issues and values of the moment in which they live. Its protection through the regulation of intellectual property rights is characterized by temporality and territoriality. The knowledge of this matter is vital for its defence in an environment presided over by the globalisation and delocalisation of companies. Our unique innovative proposal is introduced in the subject of Information Law, using the audiovisual product to study its regulation. The quantifiable results are gamified: logbook, script, playlist, audiovisual product, peer review rubric. The innovation ends with a simulation of reality, the transmission of the right, for insertion in the YouTube platform. The study of the proposed innovation has allowed us to verify that the objectives pursued have been met and also to detect the weaknesses to be resolved in future editions.
\end{abstract}

Keywords: innovation, educational video, gamification, collaborative scaffolding, individual scaffolding, logbook, play-list script, peer evaluation.

\footnotetext{
Resumen

Uno de los cambios que introduce la Declaración de Bolonia se produce en las metodologías docentes de enseñanza que pasan a estar orientadas a evaluar el esfuerzo del alumno, adquiriendo el profesor una mayor interacción en su formación. Cambios que España ha implementado por ser uno de los países firmantes, siguiendo esta línea, la Universitat Politècnica de València (UPV) a través de su plan estratégico UPV2020, acredita las Competencias Transversales de sus egresados, entre las que se encuentra la CT10 referida al Conocimiento de los Problemas Contemporáneos.
}

Para un egresado del Grado de Comunicación Audiovisual (GCAV), el producto audiovisual es su modo de expresar sus pensamientos e ideas sobre 
las cuestiones y valores políticos, sociales y legales del momento en el que vive. Su protección a través de la regulación del derecho de la propiedad intelectual, se caracteriza por la temporalidad y territorialidad. El conocimiento de esta materia es vital para su defensa en un entorno presidido por la globalización y deslocalización de las empresas. Nuestra propuesta de innovación es de tipo individual y se introduce en la asignatura de Derecho de la Información, utilizando el producto audiovisual para estudiar su regulación. Los resultados cuantificables están gamificados: cuaderno de bitácora, guion, escaleta, producto audiovisual, rúbrica de evaluación por pares. La innovación finaliza con una simulación de la realidad, la transmisión del derecho, para su inserción en la plataforma YouTube. El pase de la encuesta sobre la innovación nos ha permitido constatar que se han cumplido los objetivos perseguidos y también detectar las debilidades a resolver en próximas ediciones.

Palabras clave: innovación, video educativo, gamificación, andamiaje colaborativo, andamiaje individual, cuaderno de bitácora, guion, escaleta, evaluación por pares.

\section{Introducción}

Uno de los cambios que introduce la Declaración de Bolonia se produce en las metodologías docentes de enseñanza que pasan a estar orientadas a evaluar el esfuerzo del alumno, adquiriendo el profesor una mayor interacción en la formación del alumno. La docencia debe plantearse dirigiendo la mirada al alumno y a su aprendizaje, se trata de una enseñanza centrada más en el aprendizaje (volumen total de trabajo del alumno) que en la docencia (horas de clase), que requerirá del uso de nuevas metodologías enfocadas al desarrollo de destrezas, habilidades y competencias en el alumno (Vidal, 2012, pág. 261).

La Declaración de Bolonia de 19 de julio de 1999 implementa a los estudios del espacio europeo un plus en el que se describa con precisión las capacidades adquiridas por el alumno durante sus estudios que permita promover la adopción de un sistema homologable y equiparable de titulaciones superiores, con el fin de facilitar las mismas oportunidades de trabajo para todos los egresados en el Espacio Europeo de Educación Superior (EEES), fruto de un consenso alcanzado en Bolonia por 29 Gobiernos de la UE, cabe recordar que Kazajistán se incorporó posteriormente, en marzo 2010. En nuestro país se dotó al sistema educativo de enseñanza superior de los mecanismos necesarios para poder operar la trascendental transformación del sistema universitario español en su proceso de convergencia con el Espacio Europeo de Educación Superior, cumpliendo así con los compromisos adquiridos por el Gobierno al suscribir la Declaración de Bolonia y, en particular, con el mandato de llevar a cabo la adaptación de todas las enseñanzas a la nueva estructura en el año 2010. 
En esta sintonía, la Universitat Politècnica de València (UPV) en el curso académico 20132014 inició un proyecto, respaldado actualmente por el plan estratégico UPV2020, que pretende acreditar competencias, si bien, referidas a las Competencias Transversales de sus egresados. Entre estas competencias transversales encontramos la CT10 referida al Conocimiento de los Problemas Contemporáneos.

La asignatura en la que se introduce la presente innovación docente es la de Derecho de la Información, que se imparte en el Grado de Comunicación Audiovisual, en el segundo año. En el primer y segundo año del Grado las materias a tratar son de formación básica de la que participa ésta asignatura en la que se introduce al futuro egresado en el conjunto de normas que protegen la actividad informadora en su doble faceta individual y social o colectiva capaz de contribuir a la libre formación de una opinión publica plural (Domènech, 2014, pág. 37). Entre el contenido de esta asignatura se encuentra la regulación de la propiedad intelectual, caracterizada por su especificidad con respecto al resto de las regulaciones de los países integrantes de la Unión Europea (UE) y de mayor discrepancia con la regulación de los países de raíces anglosajonas (EEUU e Inglaterra, entre otros). El conocimiento y dominio de la regulación jurídica es imprescindible para un egresado en Comunicación Audiovisual (CAU) de la UPV, cuya actividad profesional ejercida en la era digital está presidida por la globalización y la deslocalización empresarial. El resultado del trabajo profesional, del egresado de la UPV en el Grado de CAU, es un producto audiovisual cuya autoría se protege a través de la propiedad intelectual. Conocer la regulación vigente en el marco de la materia de protección que otorga el derecho de la propiedad intelectual a sus creaciones y sus diferencias con el resto de las regulaciones es esencial para defender la paternidad que de las mismas les corresponde.

Al perfil creativo del alumno de CAU le es difícil entender la estructura de la norma jurídica y la asignatura de Derecho de la Información es la única del curriculum del grado con estas características, motivo por el cual encuentra mucha dificultad para aprender el contenido del material a estudiar. Ante este desapego por parte de los alumnos, incluso rechazo, hace urgente la búsqueda de una nueva propuesta que mejore el aprendizaje de la materia. Se plantea la utilización de la herramienta de la innovación docente. Innovación docente que debe ser entendida, como señaló el Profesor Eduardo Giménez, en unos de los Talleres de EUPU (Experto Universitario en Pedagogía Universitaria, UPV, 2016), siguiendo a Hanna (Hanna et Al. 1999, pág. 280), no necesariamente como un acto de creación, ello por cuanto que puede consistir en una mera adaptación, o, incluso, puede no ser algo nuevo. Pero, seguirá diciendo el Profesor, que debe suponer un cambio planificado y no accidental respecto a la situación previa. Nuestra innovación, siguiendo al Profesor, hemos de calificarla de tipo individual por estar relacionada con la asignatura y referirse a las necesidades de los estudiantes y a las preocupaciones profesionales como egresados de los mismos.

\section{Objetivos}

El aprendizaje significativo de las normas jurídicas que regulan la materia de la propiedad intelectual, coherente con la finalidad del proyecto. Nuestro punto de partida son las experiencias previas de los alumnos. Por el propio curriculum de los alumnos de CAU, cuando acuden a las clases de la asignatura ya han elaborado varios productos audiovisuales 
con anterioridad, de forma individual o colectiva. Atendiendo a la experiencia de los alumnos elaboraremos videos, micrometrajes, motions graphics (animación digital multimedia, porque crea movimiento mediante imágenes, fotografías, títulos, colores y diseños a la que generalmente se le añade una voz en of y música), scan scrip, etc, productos audiovisuales en los que se explique aquella parte del contenido de la materia que les haya correspondido por sorteo. En la consecución de nuestros objetivos nos apoyaremos en la teoría del andamiaje desarrollada por Wood, D. y Bruner, J. y Ross, G. (1976, pág. 66), de tal modo que el aprendizaje será activo sobre el conocimiento anterior y actual de los alumnos. Además, añadiremos el andamiaje colectivo, para ello el trabajo colaborativo será un elemento más a nuestra propuesta.

Ello permitirá:

1.- estudiar y analizar la problemática que regula de la materia de propiedad intelectual

2.- elaborar un producto audiovisual en el que se contenga la reorganización de los conceptos

3.- analizar y explicar los problemas actuales referidos a su temporalidad y territorialidad.

En resumen, al elaborar un producto audiovisual, destrezas con las que están familiarizados los alumnos, interiorizan, al hacerlo propio, como señala Ballestero, C (2016, pag. 59), el contenido referido a la regulación de la propiedad intelectual. Al ceder los derechos, como si se tratara de una venta, se toma conciencia del valor de la transmisión de la titularidad. También se cuestiona el uso de internet, herramienta digital, como fuente de documentación en clara referencia a la especialidad temporal y territorial de la normativa que regula el derecho de la propiedad intelectual en nuestro país.

\section{Desarrollo de la innovación}

La elaboración del producto audiovisual (micrometraje, motion grafic, etc...) es el instrumento que nos permitirá despertar el interés del alumno por conocer la regulación de la propiedad intelectual. Se utiliza un instrumento que ellos dominan para trabajar en el campo de unos conocimientos que hasta el momento no han experimentado, el ordenamiento jurídico y su relación con el mundo de la comunicación.

La materia objeto a tratar es regulación del derecho de propiedad intelectual, la protección que recibe en nuestro país un producto audiovisual, en todas sus manifestaciones y a quien corresponde dicha titularidad, de forma conjunta o individual y si ésta lo es de forma intensa, extensa o sólo de mero reconocimiento. La materia será subdividida en diez partes, cada una de ellas será atribuida a un grupo formado de integración libre y composición variable entre 4 y 8 integrantes. Formados los grupos y atribuida la materia, respetando determinadas pautas comunes, tendrán libertad para elaborar su propuesta en aquella forma que tengan por conveniente.

Las tareas comunes propuestas están gamificadas. Cada grupo tendrá que elaborar un cuaderno de bitácora, que permitirá tener constancia de cuándo, cómo y quién ha trabajado en cada sesión. Para elaborar un producto audiovisual, resultado final del trabajo, hay que desarrollar un guion que requiere el dominio del contenido de la regulación jurídica correspondiente al tema asignado al grupo. Posteriormente, se debe elaborar una escaleta, 
documento especifico del producto audiovisual, en el que gráficamente se coordina el guion elaborado (con su contenido jurídico) y elementos creativos a través de los cuales se va a comunicar el contenido deseado. No podemos desconocer, como señala Barros Bastida y Barros Morales, R (2015, pág. 26) que el acto de dibujar hace percibir detalles que pasan inadvertidos en una observación superficial. El dibujo tiene además dos valores a destacar en la formación, el primero, es instrumento esencial del pensamiento ya que obliga al análisis y al detalle; en segundo, es instrumento evaluador por excelencia, plasma lo que el alumno ha comprendido, analizado y sintetizado.

El contenido inicial se deberá repensar y reelaborar hasta transformarlo en lenguaje comunicativo para transmitir aquello que el grupo decida incluir en su producto audiovisual, bien a través de personales ficticios o reales, o, quizás, en voz en off. El producto final, la creación audiovisual, deberá presentarse al gran grupo, a través de una exposición pública que podrá consistir en una mera locución verbal, o auxiliada por cualquier recurso tecnológico, Power Point, Prezzi, etc.... que será grabada dando lugar a un nuevo producto audiovisual conteniendo la totalidad de las exposiciones. Cada grupo firmara un documento de cesión de sus derechos del producto audiovisual, como si de una venta se tratara, para autorizar que dicho producto pueda comunicarse públicamente a través de la plataforma YouTube, dando lugar a un archivo digital con una URL especifica. Por último, cada alumno evaluara a través de una rubrica a uno de los compañeros del mismo grupo, asignado de forma aleatoria.

El proceso, o iter, vendrá determinado por las siguientes etapas:

En primer lugar, a través de la fórmula de lección magistral, se dan unas directrices de las peculiaridades de la regulación en nuestro país, destacando las diferencias con el resto de países sobre todo los de nuestro entorno.

En segundo lugar, se propone la constitución de los grupos integrados por entre 4 y 8 alumnos y se nombra un representante. Por sorteo se atribuya la materia a los grupos.

Posteriormente, cada grupo decide como elaborara su trabajo. Se busca información adicional. Se elabora el cuaderno de bitácora, el guion, la escaleta. Además, se decide si en dicho trabajo se incluirá música y, en su caso, de qué tipo. También cómo será la exposición al gran grupo.

Cuando los grupos tienen ya elaborado el guion se reúnen los delegados de cada grupo con el profesor para unificar criterios sobre vocabulario jurídico, evitando inducir a cualquier

error, y se perfilan los contenidos soslayando la posible duplicidad en las exposiciones y que alguna cuestión quede sin considerar por sesgos de exposición.

Se expone ante el gran grupo el tema asignado, por tiempo aproximado de 15 minutos, incluida la proyección del producto audiovisual elaborado. Se suscribe un documento de cesión de derechos de la producción audiovisual para poderlo comunicar públicamente a través de la plataforma YouTube. Se entrega el material producido para su elaboración: el cuaderno de bitácora, la escaleta, el documento de cesión de derechos y cualquier otro que el 
grupo considere de interés para documentar el proceso. Además, a través de una rúbrica se evalúa de forma aleatoria a un integrante del grupo.

Al finalizar las exposiciones se propuso la participación en una encuesta, a través del formulario google encuestas en la URL https:/goo.gl/forms/g87kDfXMyTDO3ofV2, manteniendo el anonimato de los participantes. Con el fin de valorar la experiencia y aprender de la crítica, introduciendo aquellas modificaciones en los puntos débiles detectados.

\section{Resultados}

Es evidente que una de las fuentes de información se encuentra en el profesor, además, de la documentación que pueda obtenerse a través de cauces tradicionales analógicos y de cauces

Tabla 1. Cronografia

\begin{tabular}{|c|c|c|c|c|c|c|c|c|c|c|c|c|c|c|c|c|c|c|}
\hline \multirow{2}{*}{ Actividad } & \multirow[b]{2}{*}{$\begin{array}{l}\text { Respon- } \\
\text { sable }\end{array}$} & \multicolumn{17}{|c|}{ Tiempo (horas) } \\
\hline & & 1 & 2 & 3 & 4 & 5 & 6 & 7 & 8 & 9 & $\begin{array}{l}1 \\
0\end{array}$ & $\begin{array}{l}1 \\
1\end{array}$ & $\begin{array}{l}1 \\
2\end{array}$ & $\begin{array}{l}1 \\
3\end{array}$ & $\begin{array}{l}1 \\
4\end{array}$ & $\begin{array}{l}1 \\
5\end{array}$ & $\begin{array}{l}1 \\
6\end{array}$ & $\begin{array}{l}1 \\
7\end{array}$ \\
\hline $\begin{array}{l}\text { 1.-Exposición professor } \\
\text { (Lección Magistral) }\end{array}$ & Profesor & & & & & & & & & & & & & & & & & \\
\hline $\begin{array}{l}\text { 2.- Realización del Proyecto } \\
\text { (Guion y Escaleta) }\end{array}$ & Alumnos & & & & & & & & & & & & & & & & & \\
\hline $\begin{array}{l}\text { 3.- Unificación criterios y } \\
\text { delimitación sesgos }\end{array}$ & $\begin{array}{l}\text { Profes. y } \\
\text { Alumnos }\end{array}$ & & & & & & & & & & & & & & & & & \\
\hline 4- Posproducción proyecto. & Alumnos & & & & & & & & & & & & & & & & & \\
\hline $\begin{array}{l}\text { 5- Exposic. y Presentación } \\
\text { Cesión derechos y Rúbrica }\end{array}$ & Alumnos & & & & & & & & & & & & & & & & & \\
\hline
\end{tabular}

novedosos digitales (buscadores especializados, YouTube, etc...). Si atendemos al origen de las evidencias cuantitativas tenemos tres grupos, enumerados por el orden en el que aparecen en el iter de implementación de la actividad, son aquellas elaboradas por los grupos formados por generación espontánea, aquella correspondiente al gran grupo y la individual. Las evidencias cuantitativas elaboradas por cada uno de los grupos son : cuaderno de bitácora, guion, escaleta, producto audiovisual y el documento de cesión de derechos para subir el producto audiovisual a la plataforma YouTube, creando archivos para cada uno de ellos y juntos una lista de difusión, a título de ejemplo una de las direcciones de uno de los videos subido es https://youtu.be/xQbEuMkPz98. La evidencia cuantitativa elaborada por el gran grupo se corresponde con la grabación de la totalidad de las exposiciones de los grupos que como documento gráfico permitirá a los alumnos ver la evolución y el desarrollo del grupo desde el momento de la grabación hasta aquel en el que se consulte su contenido. Y, por último, la evidencia individual consistente en completar una rúbrica que se facilita para la evaluación de un integrante del grupo elegido al azar.

Esta propuesta de innovación cuenta con dos ediciones desarrolladas en dos cursos académicos correlativos. En la segunda edición nos vimos sorprendidos por la solicitud por parte de algunos alumnos de material técnico para poder elaborar productos de calidad. 
Demanda que nos sorprendió, pero habitual en otras asignaturas del curriculum del Grado. En un primer momento tuvimos algunos problemas para poder atender la demanda de los alumnos por no constar dicha necesidad en el calendario de préstamos de los equipos de titularidad institucional. Pero con la colaboración de la Dirección Académica del Grado se pudo realizar el préstamo de los equipos técnicos por parte de la Institución. Esta solicitud de demanda de material técnico por parte de algunos grupos nos puso sobre aviso sobre la calidad de los productos que pudimos observar en los trabajos presentados por los grupos que los habían solicitado. El próximo curso académico tenemos prevista una nueva edición de la presente innovación y hemos incluido la reserva de la cesión de varios equipos técnicos, ajustada al calendario de dicha actividad, entre las previsiones de cesión de material técnico para la asignatura de Derecho de la Información solicitadas a la institución para el primer cuatrimestre del próximo curso académico 2019-2020.

Una vez concluida la actividad, se planteó a los alumnos una encuesta. Del contenido de la misma podemos destacar que los alumnos prefieren la evaluación de los contenidos de la asignatura de Derecho de la Información a través de trabajos prácticos sobre cualquier otra forma de evaluación y valoran positivamente el trabajo realizado que les ha servido para entender mejor la materia de la protección del derecho de propiedad intelectual en España.

Otro de los temas que resulta relevante en la encuesta es que los alumnos han trabajado sobre las especificidades de la materia de propiedad intelectual referida a la temporalidad y espacialidad de la materia de propiedad intelectual, dentro del curriculum de la asignatura de Derecho de la Información, que conlleva la necesidad de atender en todo momento a las vicisitudes la vigencia de su regulación en un territorio concreto.

En la última pregunta de la encuesta se pedía una valoración personal de la experiencia y se pudo comprobar que los alumnos valoran positivamente el trabajar en grupo en el aula, sin que dicha colaboración sea necesario que se realice fuera del aula.

Pero la innovación no fue entendida por todos los alumnos de igual forma, como aspectos a mejorar en próximas ediciones, deberemos añadir la elaboración de una guía en la que se explique de forma más detallada el iter y las actividades a realizar que deberá facilitarse a los alumnos al iniciarse la actividad.

También fue objeto de crítica la rúbrica de evaluación por pares. Al elaborar dicha rúbrica nuestro interés se centró en la concienciación del trabajo colaborativo y no en la corruptela de dividir el trabajo de solo uno de los integrantes del grupo entre el número que lo forman. Entendemos que nos corresponde repensar la fórmula para concienciar a los alumnos sobre la cantidad y calidad del trabajo personal, de cada uno de los integrantes en el grupo, como aportación al producto final. Esto es, el trabajo colaborativo.

\section{Conclusiones}

1.- La Declaración de Bolonia, suscrita entre otros países por España, cambia las metodologías docentes de enseñanza que estarán orientadas a evaluar el esfuerzo del alumno, adquiriendo el profesor una mayor interacción con la formación del alumno. 
2.- La Universitat Politécnica de València (UPV) en el curso académico 2013-2014 inició un proyecto, respaldado actualmente por el plan estratégico UPV2020, que pretende acreditar competencias, si bien, referidas a las Competencias Transversales de sus egresados. Entre estas competencias transversales encontramos la CT10 referida al Conocimiento de los Problemas Contemporáneos.

3.- Para un egresado del Grado de Comunicación Audiovisual, el producto audiovisual es su modo de expresar sus pensamientos e ideas sobre las cuestiones y valores políticos, sociales y legales del momento en el que vive. La regulación legal de los derechos que recaen sobre el producto audiovisual se caracteriza por su temporalidad y territorialidad y su conocimiento es vital para la defensa de los derechos del egresado de la UPV en el Grado de CAU autor de la misma

4.- En la era digital no podemos ignorar la importancia del producto audiovisual, en cualquiera de sus manifestaciones (motions grafics, script cast, etc...)

5.- Se trata de una innovación docente de tipo individual, relacionada con la asignatura Derecho de la Información, vinculada a las necesidades de los estudiantes y a sus preocupaciones como futuros egresados.

6.- A través de la innovación se estudia la protección del derecho de la propiedad intelectual, detectando la especialidad de su regulación, su especialidad temporal y espacial, relevante en esta época de globalización y deslocalización empresarial.

7.- La innovación finaliza con una simulación de la realidad, la transmisión del derecho de propiedad intelectual, como si de una venta se tratara, para su comunicación pública a través de la plataforma digital YouTube.

8.- La encuesta elaborada nos ha permitido constatar que se han cumplido los objetivos perseguidos y también a detectar las debilidades a resolver en próximas ediciones.

\section{Referencias}

Libro:

DOMÈNECH MARTÍNEZ. G. (2014). Derecho de la información. Publixed. España.

\section{Revistas:}

BALLESTEROS REGAÑA, C. (2016). "Los medios audiovisuales: funciones didácticas y principios metodológicos para su integración en los procesos de enseñanza y aprendizaje". En Revista internacional de investigación e innovación educativa, págs. 58-70.

BARROS BASTIDA, C. y BARROS MORALES, R. (2015). "Los medios audiovisuales y su influencia en la educación desde alternativas de análisis". En Revista multidisciplinar de la Universidad de Cienfuegos, volumen 7, núm. 3, Septiembre- Diciembre. Cuatro Caminos. Cienfuegos. Cuba, págs. 58-70. 
VIDAL PARDO, C. (2012). "El espacio europeo de educación superior y su implantación en las universidades española”. Revista Catalana de Dret Públic, núm. 44, págs. 253-283.

WOOD, D., BRUNER, J., y ROSS, G. (1976). "El papel de la tutoría en la resolución de problemas". Revista de psicología infantil y psiquiatría y disciplinas aliadas, núm. 17, págs. 89-100.

\section{Páginas webs:}

http://eees.umh.es/contenidos/Documentos/DeclaracionBolonia.pdf (consulta marzo 2019)

https://www.boe.es/boe/dias/2010/07/03/pdfs/BOE-A-2010-10542.pdf (consulta marzo 2019)

http://www.upv.es/contenidos/COMPTRAN/ (consulta marzo 2019) 